\title{
Nitric Oxide-Donating Statins Upgrade the Benefits of Lipid-Lowering in Vascular Inflammation by Desensitizing Neutrophil Activation
}

\author{
Editorial to: "Nitric Oxide-donating atorvastatin attenuates neutrophil recruitment during \\ vascular inflammation independent of changes in plasma cholesterol” by R. Baetta et al.
}

\author{
Maik Drechsler • Oliver Soehnlein • Christian Weber
}

Published online: 26 February 2013

(C) Springer Science+Business Media New York 2013

Cardiovascular events are the leading cause for morbidity and mortality in Western societies. Clinical manifestations such as myocardial infarction and stroke mainly rely on the development and progression of atherosclerosis, which, in terms of identifying promising therapeutic targets, requires detailed understanding of its pathophysiology and underlying cellular as well as molecular mechanisms [1,2]. Atherosclerosis has widely been accepted to be a chronic inflammatory disease of the arterial wall $[1,2]$. Initially promoted by multifaceted parameters such as modified low density lipoprotein (LDL) or altered flow, it is characterized by endothelial dysfunction. Activation of endothelial cells subsequently launches a cascade of selfamplifying inflammatory processes such as expression of chemokines, cytokines and adhesion molecules, all of which then contribute to leukocyte activation, adhesion, arrest and transmigration [3]. Besides lymphocytes, which can be regularly detected in atherosclerotic plaques $[1,3]$, monocytes are

M. Drechsler $(\bowtie) \cdot$ O. Soehnlein $\cdot$ C. Weber $(\bowtie)$

Institute for Cardiovascular Prevention,

Ludwig-Maximilians-University Munich, Pettenkoferstr. 9,

80336 Munich, Germany

e-mail: maik.drechsler@med.uni-muenchen.de

e-mail: christian.weber@med.uni-muenchen.de

O. Soehnlein

Department of Pathology, Academic Medical Center Amsterdam,

University of Amsterdam, Amsterdam, the Netherlands

C. Weber

DZHK (German Centre for Cardiovascular Research),

Partner site Munich Heart Alliance, Munich, Germany

C. Weber

Cardiovascular Research Institute Maastricht,

Maastricht University, Maastricht, the Netherlands appreciated to be the most abundant subset to enter atherosclerotic lesions already in early stages and also during the course of lesion progression [3]. Hence, lesion growth is primarily sustained by constant influx of classical monocytes [4, 5]. Once monocytes have entered the lesion they differentiate into macrophages and after uptake of oxidized LDL into foam cells. However, throughout the past years it has become evident that a so far under-appreciated leukocyte subset, namely the polymorphnuclear neutrophil, is crucially involved in early atherosclerotic development [6, 7]. Lately, refined staining techniques allowed for sensitive detection of neutrophils in murine and human atherosclerotic plaque specimens. The use of antibodies to Ly6G specifically expressed on mouse neutrophils enabled the detection in early lesions as well as in ruptureprone atherosclerotic plaques [7, 8]. Unlike other leukocyte subsets, neutrophils could be identified only in rare numbers, which might be the reason why their ability to orchestrate atherosclerosis development has been considered controversial for decades. Initial implications for neutrophil-driven proatherogenic functions stem from mouse models with neutrophilia, due to deficiency in either the neutrophilhomeostasis regulating chemokine receptor CXCR4 or the hematopoietic interferon regulatory factor 8 (IRF8). Both display a massive expansion of circulating neutrophils accompanied by significantly increased atherosclerotic lesion sizes [9, 10]. Another study demonstrates that circulating neutrophil counts directly correlate with the extent of atherosclerosis and depletion of neutrophils during early stages widely protects from lesion formation due to impaired accumulation of monocytes and macrophages [7], thus being in line with observations made in microvascular models of inflammation [11, 12]. Recent work identified mechanisms underlying neutrophilmediated atherogenesis thereby proving that neutrophil- 
derived cathelicidin (human: LL37; mouse: CRAMP) is released from emigrated neutrophils and reversely transported across the endothelium, thus mediating adhesion of classical monocytes via formylated peptide receptor 2 (FPR2) [13]. In line, atherosclerotic mice deficient of CRAMP displayed reduced lesion sizes [14]. Hence, neutrophil-dependent inflammatory processes shall be taken into account when designing tools for future treatment of atherosclerosis.

Statins are regularly used to reduce plasma cholesterol, thus interfering with one main risk factor for atherosclerosis but additionally display beneficial effects by reduction of interleukin-1 $\beta$ [15] and CD40L [16]. Furthermore they were reported to impair oxLDL mediated expression of endothelial adhesion molecules [17] and improve endothelial function by restoration of the nitric oxide (NO) production [18]. However, under severe hypercholesterolemia conventional statins are not eligible to completely restore endothelial function and NO synthesis $[19,20]$. In recent years a new compound class evolved. Further to the lipid lowering effect by inhibiting the HMG-CoA reductase, NO-donating statins can additionally provide bioactive $\mathrm{NO}$ thereby promoting anti-thrombotic and anti-inflammatory properties [21-23]. In this regard, a recent study provided evidence that NO-donating atorvastatin (NCX6560) enhances the beneficial effects of conventional atorvastatin in a mouse model of accelerated atherosclerosis [24] including decreased lesion formation, reduced MMP-2 expression and IL-6 serum levels. However, although NOdonating statins might expand the positive features of the conventional ones in atheroprotection, a mechanistic link on cellular level could not be provided so far.

In this issue of Cardiovascular Drugs and Therapy, Baetta et al. investigate the impact of NO-donating atorvastatin (NCX6560) and conventional atorvastatin on neutrophils during vascular inflammation independently of plasma cholesterol. By using a model of perivascular collar placement around the carotid artery of New Zealand White rabbits, which were treated with either atorvastatin or NCX6560, they observed significantly diminished numbers of neutrophils within the carotid artery of animals receiving NO-donating atorvastatin [25]. Thus, acute neutrophil recruitment as a consequence of vascular injury induced by disturbed flow could be reduced by providing bioavailable NO. As animals received no high fat diet (HFD) and treatment with conventional atorvastatin resulted in an altered neutrophil recruitment, this study clearly indicates an additional beneficial effect of the NO, provided by a statin independent of its impact on plasma cholesterol levels [25]. To gain further insights into the cellular responses induced by the NO donating properties of NCX6560 which could explain the reduced neutrophil accumulation after collar placement, the authors performed several in vitro experiments. From these experiments it became evident that neutrophil activation is down-regulated by NO-donating statins as cells became less susceptible to IL8-induced chemotaxis.
Furthermore, TNF $\alpha$ or fMLP-induced release of IL 8 by either HUVECs or neutrophils was decreased when cells were pretreated with atorvastatin or NCX6560. Worth mentioning, the observed reduction was even more remarkable for the NOdonating statin [25]. As neutrophil function in atherosclerosis may primarily be observed as a subsequent recruitment of inflammatory monocytes, Baetta et al. analyzed the ability of cell free supernatants of fMLP-stimulated neutrophils pretreated with either atorvastatin or NCX6560 to induce monocyte transmigration. In line, supernatants from neutrophils pretreated with NCX6560 displayed reduced monocyte transmigration compared to the respective control as well as atorvastatin, indicating a beneficial effect of $\mathrm{NO}$ on the release of monocyte attracting factors [25].

The study by Baetta et al. nicely demonstrates how NOdonating statins provide additional beneficial effects under conditions of endothelial dysfunction not only on endothelial cells but also on neutrophils. Beside the well described serum cholesterol lowering effects of atorvastatin, NCX6560 reduces neutrophil recruitment by providing bioavailable $\mathrm{NO}$ which impairs neutrophil activation and thereby might synergistically effect the subsequent recruitment of monocytes. However, one carefully has to consider that neutrophils are primarily important in early lesion formation. In contrast, depletion of neutrophils in mice with advanced atherosclerosis did not show any impact on atheroprogression [7]. Translated into a potential therapeutic approach in humans, that would ultimately mean that treatment should be initiated early in life. Although, the role of neutrophils in the destabilization of atherosclerotic plaques is still under debate, this could be a more feasible target for NO-donating statins. Furthermore, myocardial infarction has been proven to accelerate atherosclerosis [26], a shematopoietic stem cells are liberated from the bone marrow to undergo extramedullary proliferation and differentiation within the spleen thus increasing the number of circulating myeloid cells such as classical monocytes and neutrophils, both of which easily infiltrate atherosclerotic lesions [26]. These findings might explain why survivors of acute coronary syndrome are more susceptible for recurrent cardiovascular events. It would be interesting to investigate whether application of NO-donating statins directly after acute myocardial infarction could be beneficial by down-regulating the activation status of neutrophils. However, one has to keep in mind that neutrophils possess an ambivalent role in vascular injury as occurs during interventional stent implantation. Using a mouse-model of endothelial denudation, neutrophilderived cathelicidin promoted re-endothelization thereby limiting neointima-formation [27]. In line, mice that obtained stents coated with cathelicidin displayed significantly reduced in-stent stenosis. Similarly, evidence for an angiogenic, tissueregenerating effect of neutrophils is accumulating [28]. Hence, in such conditions reduction of neutrophil activation may prove counterproductive. 
Taken together, addressing neutrophil activation status by NO-donating statins appears to be a promising therapeutic approach, which however needs to be substantiated in future investigations focusing on specific interventional approaches.

\section{References}

1. Hansson GK. Inflammation, atherosclerosis, and coronary artery disease. N Engl J Med. 2005;352:1685-95.

2. Weber C, Noels H. Atherosclerosis: current pathogenesis and therapeutic options. Nat Med. 2011;17:1410-22.

3. Weber C, Zernecke A, Libby P. The multifaceted contributions of leukocyte subsets to atherosclerosis: lessons from mouse models. Nat Rev Immunol. 2008;8:802-15.

4. Swirski FK, Pittet MJ, Kircher MF, Aikawa E, Jaffer FA, Libby P, et al. Monocyte accumulation in mouse atherogenesis is progressive and proportional to extent of disease. Proc Natl Acad Sci U S A. 2006;103:10340-5.

5. Soehnlein O, Drechsler M, Döring Y, Lievens D, Hartwig H, Kemmerich K et al. Distinct function of chemokine receptor axes in the atherogenic mobilization and recruitment of classical monocytes. EMBO Mol Med. 2013. doi:10.1002/emmm.201201717.

6. Drechsler M, Doring Y, Megens RT, Soehnlein O. Neutrophilic granulocytes - promiscuous accelerators of atherosclerosis. Thromb Haemost. 2011;106:839-48.

7. Drechsler M, Megens RTA, van Zandvoort M, Weber C, Soehnlein O. Hyperlipidemia-Triggered Neutrophilia Promotes Early Atherosclerosis. Circulation. 2010;122:1837-45.

8. van Leeuwen M, Gijbels MJ, Duijvestijn A, Smook M, van de Gaar MJ, Heeringa P, et al. Accumulation of myeloperoxidasepositive neutrophils in atherosclerotic lesions in LDLR-/- mice. Arterioscler Thromb Vasc Biol. 2008;28:84-9.

9. Zernecke A, Bot I, Djalali-Talab Y, Shagdarsuren E, Bidzhekov K, Meiler S, et al. Protective role of CXC receptor 4/CXC ligand 12 unveils the importance of neutrophils in atherosclerosis. Circ Res. 2008;102:209-17.

10. Doring Y, Soehnlein O, Drechsler M, Shagdarsuren E, Chaudhari SM, Meiler S, et al. Hematopoietic interferon regulatory factor 8deficiency accelerates atherosclerosis in mice. Arterioscl Thromb Vasc Biol. 2012;32:1613-23.

11. Soehnlein O, Lindbom L, Weber C. Mechanisms underlying neutrophil-mediated monocyte recruitment. Blood. 2009;114:461323.

12. Soehnlein O, Zernecke A, Eriksson EE, Rothfuchs AG, Pham CT, Herwald $\mathrm{H}$, et al. Neutrophil secretion products pave the way for inflammatory monocytes. Blood. 2008;112:1461-71.

13. Wantha S, Alard JE, Megens RT, van der Does AM, Doring Y, Drechsler $\mathrm{M}$ et al. Neutrophil-derived cathelicidin promotes adhesion of classical monocytes. Circ Res 2013.

14. Doring Y, Drechsler M, Wantha S, Kemmerich K, Lievens D, Vijayan S, et al. Lack of neutrophil-derived CRAMP reduces atherosclerosis in mice. Circ Res. 2012;110:1052-6.
15. Waehre T, Yndestad A, Smith C, Haug T, Tunheim SH, Gullestad $\mathrm{L}$, et al. Increased expression of interleukin-1 in coronary artery disease with downregulatory effects of HMG-CoA reductase inhibitors. Circulation. 2004;109:1966-72.

16. Pignatelli P, Sanguigni V, Lenti L, Loffredo L, Carnevale R, Sorge R, et al. Oxidative stress-mediated platelet CD40 ligand upregulation in patients with hypercholesterolemia: effect of atorvastatin. J Thromb Haemost. 2007;5:1170-8.

17. Li D, Chen H, Romeo F, Sawamura T, Saldeen T, Mehta JL. Statins modulate oxidized low-density lipoprotein-mediated adhesion molecule expression in human coronary artery endothelial cells: role of LOX-1. J Pharmacol Exp Ther. 2002;302:601-5.

18. Laufs U, Gertz K, Huang P, Nickenig G, Bohm M, Dirnagl $\mathrm{U}$, et al. Atorvastatin upregulates type III nitric oxide synthase in thrombocytes, decreases platelet activation, and protects from cerebral ischemia in normocholesterolemic mice. Stroke. 2000;31:2442-9.

19. John S, Schneider MP, Delles C, Jacobi J, Schmieder RE. Lipidindependent effects of statins on endothelial function and bioavailability of nitric oxide in hypercholesterolemic patients. Am Heart J. 2005;149:473.

20. Ling MC, Ruddy TD, deKemp RA, Ukkonen H, Duchesne L, Higginson L, et al. Early effects of statin therapy on endothelial function and microvascular reactivity in patients with coronary artery disease. Am Heart J. 2005;149:1137.

21. Ongini E, Impagnatiello F, Bonazzi A, Guzzetta M, Govoni M, Monopoli A, et al. Nitric oxide (NO)-releasing statin derivatives, a class of drugs showing enhanced antiproliferative and antiinflammatory properties. P Natl Acad Sci USA. 2004;101:8497502 .

22. Rossiello MR, Momi S, Caracchini R, Giannini S, Guglielmini G, Monopoli A, et al. A novel nitric oxide-releasing statin derivative exerts an antiplatelet/antithrombotic activity and inhibits tissue factor expression. J Thromb Haemost. 2005;3:2554-62.

23. Momi S, Impagnatiello F, Guzzetta M, Caracchini R, Guglielmini G, Olivieri R, et al. NCX 6560, a nitric oxide-releasing derivative of atorvastatin, inhibits cholesterol biosynthesis and shows antiinflammatory and anti-thrombotic properties. Eur J Pharmacol. 2007;570:115-24.

24. Momi S, Monopoli A, Alberti PF, Falcinelli E, Corazzi T, Conti V, et al. Nitric oxide enhances the anti-inflammatory and antiatherogenic activity of atorvastatin in a mouse model of accelerated atherosclerosis. Cardiovasc Res. 2012;94:428-38.

25. Baetta R, Granata A, Miglietta D, Oliva F, Arnaboldi L, Bonomo A et al. Nitric oxide-donating atorvastatin attenuates neutrophil recruitment during vascular inflammation independent of changes in plasma cholesterol. Cardiovasc Drugs Ther. 2013;27:this issue.

26. Dutta P, Courties G, Wei Y, Leuschner F, Gorbatov R, Robbins CS, et al. Myocardial infarction accelerates atherosclerosis. Nature. 2012;487:325-9.

27. Soehnlein O, Wantha S, Simsekyilmaz S, Doring Y, Megens RT, Mause SF, et al. Neutrophil-derived cathelicidin protects from neointimal hyperplasia. Sci Trans1 Med. 2011;3:103ra98.

28. Christoffersson G, Vagesjo E, Vandooren J, Liden M, Massena S, Reinert RB, et al. VEGF-A recruits a proangiogenic MMP-9delivering neutrophil subset that induces angiogenesis in transplanted hypoxic tissue. Blood. 2012;120:4653-62. 